\title{
Combined vitamin D and calcium supplementation in vitamin D inadequate patients with urolithiasis: Impact on hypercalciuria and de novo stone formation
}

\author{
Charles Hesswani, BSc; ${ }^{*}$ Yasser A. Noureldin, MD, MSc, PhD; ${ }^{*+}$ Mohamed A. Elkoushy, MD, MSc, PhD, ${ }^{*{ }_{5}}$ \\ Sero Andonian, MD, MSc, FRCSC, FACS
}

*Division of Urology, McGill University Health Centre, McGill University, Montreal, QC; 'Department of Urology, Benha University Hospital, Benha University, Benha, Egypt; §Department of Urology, Suez Canal University, Ismailia, Egypt

Cite as: Can Urol Assoc J 2015;9(11-12):403-8. http://dx.doi.org/10.5489/cuaj.3332

Published online December 14, 2015.

\section{Abstract}

Introduction: We examined the effect of combined vitamin D and calcium supplementation (VDCS) on urinary calcium excretion and de novo stone formation in vitamin D inadequate (VDI) urolithiasis patients.

Methods: We retrospectively reviewed the data of VDI patients (serum 25-hydroxyvitamin D $<75 \mathrm{nmol} / \mathrm{L}$ ) followed at a tertiary stone centre between September 2009 and December 2014. VDI patients with history of urolithiasis, who were placed on VDCS for abnormal bone mineral density or hyperoxaluria, were included. Hypercalciuric patients and patients on thiazide diuretics were excluded. Metabolic stone workup and two 24-hour urine collections were performed before and after VDCS.

Results: In total, we included 34 patients, with a mean age of 54.8 years and a mean body mass index of $25.7 \mathrm{~kg} / \mathrm{m}^{2}$. After VDCS, there was a significant increase in the mean serum 25-hydroxyvitamin D (52.0 vs. $66.4 \mathrm{nmol} / \mathrm{L}, p<0.001)$ and the mean urinary calcium excretion ( 3.80 vs. $5.64 \mathrm{mmol} / \mathrm{d}, p<0.001)$. Eight $(23.5 \%)$ patients developed de novo hypercalciuria. After a median followup of 39 (range: $7-60$ ) months, 50\% of hypercalciuric patients developed stones compared with $11.5 \%$ of non-hypercalciuric patients $(p=0.038)$.

Conclusion: This study showed a significant effect of combined VDCS on mean urinary calcium excretion, de novo hypercalciuria, and stone development in VDI patients with history of urolithiasis. Therefore, VDI urolithiasis patients receiving VDCS are advised to have monitoring with 24-hour urine collections and imaging studies. Although small, our sample size was good enough to validate the statistical outcomes. Prospective studies are needed to confirm these results.

\section{Introduction}

Vitamin D inadequacy (VDI) is an endemic condition affecting about 1 billion people worldwide. ${ }^{1,2}$ Inadequate serum 25-hydroxyvitamin D [25(OH)VD] adversely affects bone strength and various health conditions. ${ }^{3}$ In fact, VDI is present in as many as 4 out of 5 patients with urolithiasis, with $20 \%$ of them being hypercalciuric. ${ }^{4}$ Despite the role of calcium in maintaining good health and preventing bone loss, urologists are reluctant to treat VDI in patients with history of urolithiasis due to concerns for increased urinary calcium excretion and thus increased risk of urolithiasis in this high-risk population. Although several studies have examined the relationship between vitamin D supplementation (VDS) and hypercalciuria, evidence remains limited and contradictory on whether a correlation exists between both conditions. Some studies detected an increase in urinary calcium excretion and kidney stone development after VDS, whereas others did not. ${ }^{5-10}$

Due to the unknown effects of vitamin D and calcium supplementation (VDCS) on the risk of developing hypercalciuria and subsequent de novo stone formation, we examined the impact of maintenance doses of vitamin D (VD) and calcium on urinary calcium excretion and de novo stone development in VDI patients with a history of urolithiasis. Our hypothesis is that urinary calcium excretion would significantly increase, such that some patients would fall in the hypercalciuria range. Furthermore, the rate of de novo stone development would be greater in these hypercalciuric patients.

\section{Methods}

After obtaining institutional ethics approval, we retrospectively reviewed the data of VDI patients with a history of urolithiasis at a tertiary stone centre between September 2009 and December 2014. Patients with serum $25(\mathrm{OH})$ VD levels below $75 \mathrm{nmol} / \mathrm{L}$ and history of urolithiasis who received VDCS for VDI, osteoporosis (Z score $<-2.5$ SD) or hyperoxaluria (urinary oxalate $>480 \mathrm{umol} / \mathrm{L}$ ) were included in the study. ${ }^{11}$ Serum $25(\mathrm{OH})$ VD levels were measured by high- 
Hesswani et al.

pressure liquid chromatography-tandem mass spectrometry (AB SCIEX, Foster City, CA). We excluded patients (1) with primary hyperparathyroidism (HPT); (2) without pre- and post-VDCS metabolic stone workup; (3) with hypercalciuria (urinary calcium excretion $>300 \mathrm{mg} / \mathrm{d}$ for men, $>275 \mathrm{mg} / \mathrm{d}$ for women) prior to VDCS; (4) on thiazide diuretic prior to VDCS; and (5) pregnant women (Fig. 1). We also reviewed patient charts, including imaging studies.

Metabolic stone workup included two 24-hour urine collections prior to and 3 months post VDCS. When there was discrepancy between the two 24-hour urine collections, the collection with the higher urinary calcium was chosen as the representative sample. The urinary parameters included volume, $\mathrm{pH}$, 24-hour excretion of creatinine, calcium, sodium, phosphate, uric acid, citrate, oxalate, potassium, magnesium, and urea nitrogen. Serum analysis included serum $25(\mathrm{OH})$ VD levels, total and normalized ionized calcium, creatinine, uric acid and parathyroid hormone (PTH). Patients with an elevated PTH underwent sestamibi parathyroid scintigraphy and neck ultrasonography to differentiate between primary and secondary HPT. Normalized ionized calcium levels were also used to differentiate between the two HPT states. Patients with primary HPT were excluded.

Patients were followed with imaging studies semi-annually. Radiology imaging and reports were used to assess the development of new stones and/or growth of pre-existing stones in comparison to pre-treatment studies. Stone development was defined as an increase in the size of previous stones and/or the development of new stones.

\section{Statistical analysis}

Descriptive data were presented in terms of numbers, percentages, medians, means and 95\% confidence intervals (Cl) of the means. Fisher's exact test was used to compare between categorical variables whereas the paired Student t-test was used to compare within-group continuous parameters pre- and post-VDCS. Linear regression model was used to examine the relationship between changes in serum $25(\mathrm{OH}) \mathrm{VD}$ and changes 24 -hour urinary calcium excretion. A two-sided level of significance $p<0.05$ was adopted for all tests. All data analysis was performed using IBM Statistical Package for Social Sciences for Macintosh, v.21 (SPSS, Cary, NC).

\section{Results}

In total, we included 34 patients, with a mean age of $54.8 \pm 14.7$ years and a mean body mass index of $25.7 \pm 4.78 \mathrm{~kg} / \mathrm{m}^{2}$. Females comprised $32.4 \%$ and family history of urolithiasis was positive in $48.4 \%$ of patients. In total, $20 \%$ of patients were osteoporotic, $32.4 \%$ were hyperoxaliuric, and $53.1 \%$ were recurrent stone-formers (Table 1 ). The median VD and calcium doses were 800 (range: 4001200) IU and 1000 (range: 500-1500) mg for Ci-Cal D, 1500 (range: 500-1500) IU; and 945 (range: 315-945) mg for Osteocit+D, $800 \mathrm{IU}$ and $1000 \mathrm{mg}$ for CarbocalD and 1000 (range: 800-1428) IU and $500 \mathrm{mg}$ for generic VD (Table 1). Median VD and calcium doses supplied were 1000 (range: 400-1500) IU/day and 945 (range: 315-1500) mg/ day, respectively (Table 1 ). After a median follow-up of

Met inclusion criteria $(\mathrm{n}=61)$

1. Previous-stone formers

2. $V D$ inadequate $(<75 \mathrm{nmol} / \mathrm{L})$

3. Receiving maintenance doses of combined VD and calcium

Excluded $(n=27)$

- 10 hypercalciuric pre-VDCS

- 7 on thiazide diuretic pre-VDCS

- 7 lacking $25(\mathrm{OH})$ VD measurement pre-VDCS

- 2 lacking $25(\mathrm{OH})$ VD measurement post-VDCS

- 1 pregnant

Included in the analysis $(n=34)$

Fig. 1. Flow diagram of the inclusion and exclusion criteria. VD: vitamin D; VDCS: vitamin D and calcium supplementation. 


\begin{tabular}{lc}
\hline Table 1. Baseline characteristics & \\
\hline Parameter (n = 34) & Value \\
\hline Age (mean \pm SD) (years) & $54.8 \pm 14.7$ \\
BMI (mean \pm SD) $\left(\mathrm{kg} / \mathrm{m}^{2}\right)$ & $25.7 \pm 4.78$ \\
Female (\%) & 32.4 \\
Family history of stones (\%) & 48.4 \\
Recurrent stone formers (\%) & 53.1 \\
Daily vitamin D dose (median [range] IU) & $1000(400-1500)$ \\
Daily calcium dose (median [range], mg) & $945(315-1500)$ \\
Vitamin D and calcium medication* (\%) & \\
- Ci-Cal D (500 mg, 400 IU) & $41 \%$ \\
- Osteocit + D (315 mg, 500 IU) & $41 \%$ \\
- Carbocal D (500 mg, 400 IU) & $3 \%$ \\
- Generic VD and calcium & $15 \%$ \\
Follow-up period (months) (median [range]) & $39(7-60)$ \\
Osteoporotic (\%) & 20.1 \\
Hyperoxaluric* (\%) & 32.4 \\
\hline SD: standard deviation; BMl: body mass index; IU: international units. *Urinary oxalate \\
$>480$ umol/L.
\end{tabular}

39 months (range: $7-60$ ), serum $25(\mathrm{OH}) \mathrm{VD}$ significantly increased from 52.0 to $66.4 \mathrm{nmol} / \mathrm{L}, p<0.001$ (Table 2). Despite this significant increase, only $13(38.2 \%)$ patients in the cohort had normalized their serum 25(OH)VD levels (Table 3).

The mean serum total calcium did not change after VDCS $(p=0.568)$, whereas the mean serum ionized calcium rose significantly $(4.62 \pm 0.28$ vs. $4.88 \pm 0.36 \mathrm{mmol} / \mathrm{L}, p=0.001)$. However, no patient became hypercalcemic ( $>1.32 \mathrm{mmol} / \mathrm{L})$. The mean 24-hour urinary calcium excretion significantly increased after VDCS (3.80 vs. $5.64 \mathrm{mmol} / \mathrm{d}, p<0.001)$, and $8(23.5 \%)$ patients developed de novo hypercalciuria $(p=0.001)$ (Table 3$)$. Moreover, the mean urinary oxalate levels significantly decreased after VDCS (413 vs. $352 \mu \mathrm{mol} / \mathrm{d}, p=0.032$ ) (Table 2) and the number of hyp- eroxaliuric patients significantly decreased from 11 to 3 $(p=0.016)$ (Table 3).

No significant change was observed for the mean serum PTH levels $(p=0.987)$ after VDCS (Table 2$)$. Nine $(27.3 \%)$ patients had secondary HPT prior to VDCS (Table 3 ). The mean urinary sodium, potassium and citrate rose significantly after VDCS $(p=0.004, p=0.034$ and $p<0.001$, respectively).

Out of the $8(23.5 \%)$ patients who developed de novo hypercalciuria, 4 (50.0\%) developed new stones and/or had their stones grow in size. In contrast only $3 / 26(11.5 \%)$ non-hypercalciuric patients developed stones. The difference in de novo stone development between the 2 groups was significant ( $p=0.038$ ) (Fig. 2$)$. Four $(50.0 \%$ ) of the 8 de novo hypercalciuric patients received thiazide diuretics to decrease their urinary calcium excretion. Additionally, $9(25.7 \%)$ patients were hypernatriuric (urinary sodium excretion $>220 \mathrm{mmol} / \mathrm{d}$ ) on follow-up 24-hour collections (Table 2). The number of hypernatriuric patients was similar between hypercalciuric and non-hypercalciuric patients at follow-up ( $25 \%$ vs. $27 \%, p=0.464)$.

A linear regression analysis was generated to correlate changes in urinary calcium excretion and serum $25(\mathrm{OH})$ VD levels (Fig. 3). Although the mean urinary calcium excretion rose significantly after VDCS (Table 2), there was no significant correlation between changes in urinary calcium excretion and changes in serum 25(OH)VD levels $(r=0.032, p=0.857)$.

\section{Discussion}

Despite VDI's crucial role in calcium homeostasis, VDI stone formers are rarely prescribed VDCS due to the possibility that it may increase urinary calcium excretion and promote

\begin{tabular}{|c|c|c|c|c|}
\hline Variable & & Baseline mean $(95 \% \mathrm{Cl})$ & Follow-up mean $(95 \% \mathrm{CI})$ & $p$ value \\
\hline \multirow{6}{*}{ Serum } & $25(\mathrm{OH}) \mathrm{VD}(\mathrm{nmol} / \mathrm{L})$ & $52.0(46.2-57.8)$ & $66.4(58.3-74.6)$ & $<0.001$ \\
\hline & Total calcium (mmol/L) & $2.33(2.27-2.38)$ & $2.33(2.28-2.38)$ & 0.568 \\
\hline & Ionized calcium (mmol/L) & $1.15(1.13-1.18)$ & $1.22(1.19-1.26)$ & 0.001 \\
\hline & PTH intact (pmol/L) & $5.65(4.90-6.41)$ & $5.64(4.80-6.48)$ & 0.987 \\
\hline & Creatinine (umol/L) & $89.3(77.7-100.1)$ & $86.9(76.6-97.1)$ & 0.083 \\
\hline & Uric acid (umol/L) & $348(322-374)$ & $325(296-354)$ & 0.107 \\
\hline \multirow{8}{*}{ 24-hour urine collections } & Calcium (mmol/d) & $3.80(3.27-4.33)$ & $5.64(4.78-6.49)$ & $<0.001$ \\
\hline & Phosphorous (mmol/d) & $23.3(20.9-25.7)$ & $22.6(20.7-24.5)$ & 0.602 \\
\hline & Citrate $(\mathrm{mmol} / \mathrm{d})$ & $2.53(1.87-3.20)$ & $3.50(2.78-4.21)$ & $<0.001$ \\
\hline & Oxalate (umol/d) & $413(346-479)$ & $352(285-419)$ & 0.032 \\
\hline & Sodium (mmol/d) & $143(124-161)$ & 173 (150-197) & 0.004 \\
\hline & Uric acid (mmol/d) & $3.03(2.70-3.37)$ & $2.97(2.58-3.36)$ & 0.730 \\
\hline & Potassium (mmol/d) & $56.0(48.5-63.5)$ & $67.5(59.0-76.1)$ & 0.034 \\
\hline & Magnesium (mmol/d) & $4.68(2.92-6.44)$ & $4.11(3.10-5.13)$ & 0.580 \\
\hline
\end{tabular}


Hesswani et al.

\begin{tabular}{|c|c|c|c|c|}
\hline Variable & & Baseline no. (\%) & Follow-up no. (\%) & $p$ value \\
\hline \multirow{4}{*}{ Serum } & Inadequate vitamin $25(\mathrm{OH}) \mathrm{VD}(<75 \mathrm{mmol} / \mathrm{L})$ & $34(100)$ & $21(61.8)$ & $<0.001$ \\
\hline & Hypercalcemia (ionized calcium >1.32 mmol/L) & $0(0)$ & $0(0)$ & 0 \\
\hline & HPTH (>6.9 pmol/L) & $9(27.3)$ & $8(24.2)$ & 1.0 \\
\hline & Hyperuricemia (>353 umol/L) & $13(38.2)$ & $14(41.2)$ & 1.0 \\
\hline \multirow{7}{*}{ 24-hour urine collections } & Hypercalciuria ( $>7.5 \mathrm{mmol} / \mathrm{d}$ for $\mathrm{men} ;>6.9 \mathrm{mmol} / \mathrm{d}$ for women) & $0(0)$ & $8(23.5)$ & 0.005 \\
\hline & Hypocitraturia (<1.6 mmol/d) & $3(13.0)$ & $1(4.3)$ & 0.610 \\
\hline & Hyperoxaluria (>480 umol/d) & $11(32.4)$ & $3(8.8)$ & 0.016 \\
\hline & Hyperuricosuria (>4.4 mmol/d) & $2(5.9)$ & $3(8.8)$ & 1.0 \\
\hline & Hypernatriuria (>220 mmol/d) & $4(11.8)$ & $9(26.5)$ & 0.213 \\
\hline & Hyperkaluria (>77 mmol/d) & $4(13.8)$ & $7(24.1)$ & 0.501 \\
\hline & Hypermagnesuria (>5 mmol/d) & $9(27.3)$ & $6(18.2)$ & 0.560 \\
\hline
\end{tabular}

VD: vitamin D; VDCS: vitamin D and calcium supplementation.

urolithiasis. In the present study, after VDCS, there was a significant increase in mean serum 25(OH)VD levels and mean urinary calcium excretion. Furthermore, 8 patients $(23.5 \%)$ developed de novo hypercalciuria $(p=0.005)$.

These results are different from the findings by Leaf and colleagues, where the authors gave VD repletion therapy (ergocalciferol $50000 \mathrm{IU} /$ week) for 8 weeks without calcium supplementation. ${ }^{8}$ They found that mean 24 -hour urinary calcium excretion did not change significantly after 8 weeks. The lack of significant changes in urinary calcium excretion could be due to short-duration of therapy and lack of calcium supplementation. The median follow-up time in the present study was 39 months and all patients received calcium in addition to VD. In addition, in the former study, patients with hypercalciuria or on concomitant thiazide diuretics were not excluded and there was no mention of any patients developing hypercalciuria. In the present study, patients with hypercalciuria prior to VDCS were excluded (Fig. 1). A quarter of hypercalciuria were prescribed a thiazide diuretic. our patients $(23.5 \%)$ developed de novo hypercalciuria. In addition, 4 patients who developed de novo Finally, the current study assessed stone development

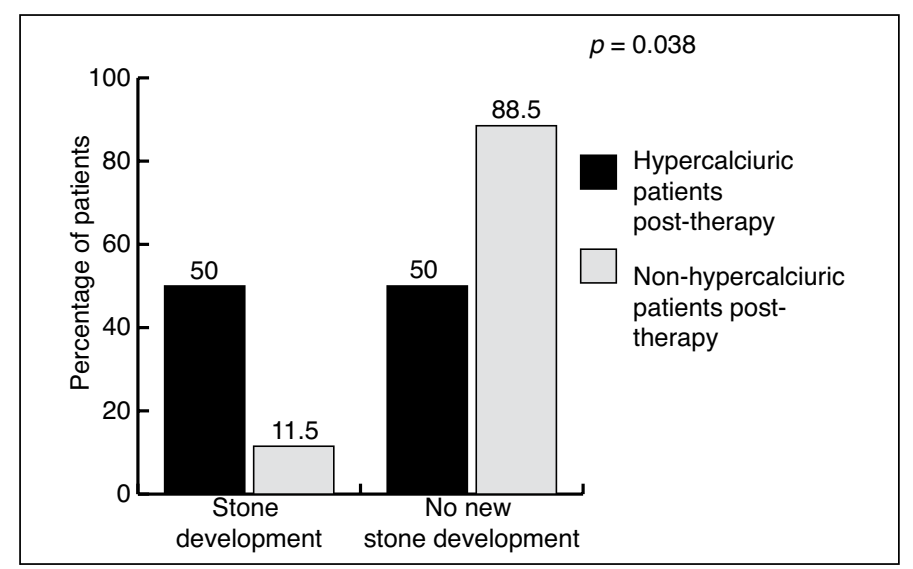

Fig. 2. De novo stone development in hypercalciuric and non-hypercalciuric patients after vitamin $\mathrm{D}$ and calcium supplementation. or growth after VDCS where all patients were followed with imaging studies.

In the present study, $50 \%$ percent of hypercalciuric patients developed stones compared with $11.5 \%$ of nonhypercalciuric patients $(p=0.038)$ (Fig. 2). These results are consistent with Jackson and colleagues' study that showed a $17 \%$ increase in de novo stone development among patients receiving VDCS (1000 mg calcium daily and 400 IU vitamin $\mathrm{D}_{3}$ daily). ${ }^{5}$

Similarly, Wallace and colleagues showed that postmenopausal women on VDCS (1000 mg calcium daily and $400 \mathrm{IU}$ vitamin $D_{3}$ daily) for an average of 7 years self-reported an increase in incidence of urolithiasis. ${ }^{6}$ Whereas the previous 2 studies exclusively enrolled postmenopausal women without a history of stones who self-reported occurrence of stones, the present study enrolled both men and women with history of urolithiasis and were followed with serial imaging studies to confirm de novo stone development. Since menopause is associated with increased urinary calcium excretion, it may have confounded the results. ${ }^{12}$

There was no correlation between changes in serum 25(OH)VD levels and urinary calcium excretion $(r=0.032, p=0.857)$ (Fig. 3). These results are consistent with the findings by Eisner and colleagues. ${ }^{7}$ Whereas the latter study used a single 24-hour urine collection, in the present study two 24-hour urine collections were used to get a better representative sampling of the urinary parameters as recommended by the Canadian Urology Association guidelines. ${ }^{13,14}$ On the other hand, the study by Berlin and colleagues found that patients with high urinary calcium excretion $(>6.0 \mathrm{mmol} / \mathrm{L})$ had significantly higher levels of serum $25(\mathrm{OH})$ VD than those with a lower urinary calcium. ${ }^{15}$ However, patients were prescribed neither VD nor calcium supplements. In comparison, all patients in the current study were supplemented with both VD and calcium, and changes in serum $25(\mathrm{OH}) \mathrm{VD}$ and urinary calcium levels were examined rather than correlated with absolute values. 


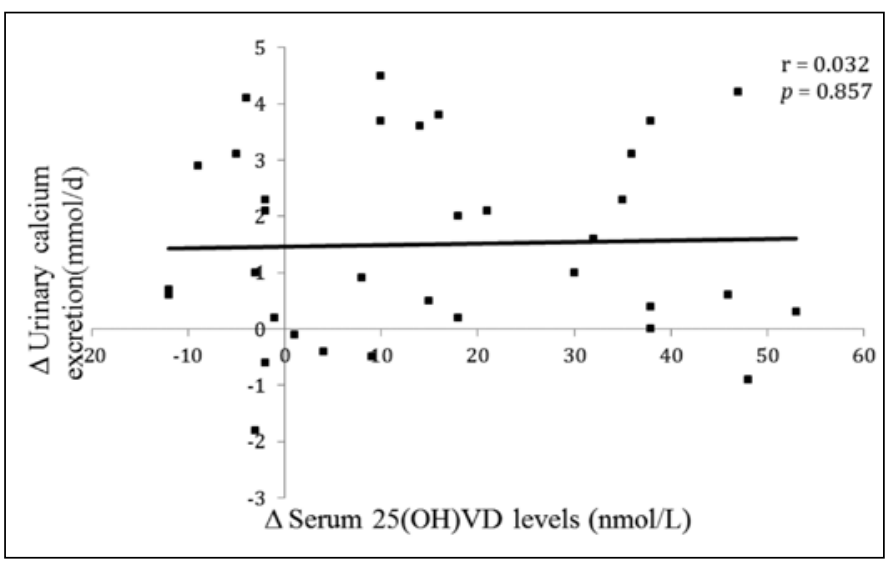

Fig. 3. Change in urinary calcium excretion versus change in serum 25 -hydroxyvitamin D post vitamin D and calcium. supplementation

In the present study, VDCS did not suppress serum PTH levels significantly $(p=0.987)$. This could be due to the fact that only $38.2 \%$ of patients normalized their $25(\mathrm{OH})$ VD levels, since VDS rather than repletion was used in the present study. Interestingly, the daily VD doses (median of $1000 \mathrm{IU}$ ) used in the current cohort were higher than Health Canada's recommended dietary allowance per day for VD (600 IU if $<70$ years old, $800 \mathrm{IU}$ if older) and calcium intake $(25 \mathrm{mmol} / \mathrm{d}$ for men, $30 \mathrm{mmol} / \mathrm{d}$ for women $){ }^{16}$ The doses used in the present study are consistent with the findings of a recent review article which recommended a daily VD intake over $1000 \mathrm{IU}$ to bring 25(OH)VD concentrations above $75.0 \mathrm{nmol} / \mathrm{L} .{ }^{17}$ The citrate content of the medications explains the significant increase in mean urinary citrate excretion $(2.53$ vs. $3.50 \mathrm{mmol} / \mathrm{d}, p<0.001)$. In addition, $19(55.8 \%)$ patients were prescribed potassium citrate to alkalinize urine and/or treat hypocitraturia. Therefore, this explains the significantly increased mean urinary potassium and citrate excretion on follow-up (Table 1).

As expected, the number of hyperoxaliuric patients decreased significantly after VDCS $(p=0.016)$. All patients were given the same advice to increase fluids $>2 \mathrm{~L} /$ day, and follow a low salt $(<2300 \mathrm{mg} /$ day $)$, low oxalate and low purine diet. Since mean urinary sodium excretion increased significantly after VDCS $(p=0.004)$ and 9 patients were hypernatriuric, urinary sodium excretion could have confounded the effects of VDCS (Table 2). However, patients who developed de novo hypercalciuria had similar urinary sodium levels compared to patients with normal urinary calcium $(p=0.4)$.

Our retrospective study includes several inherent limitations. The sample size was relatively small, but good enough to validate the statistical outcomes. In addition, intra-individual 25(OH)VD levels tended to fluctuate, and $11(32.4 \%)$ patients showed a decrease in their serum VD at their last serum measurements. Therefore, non-adherence to medications and subsequent lack of normalization of their serum
25(OH)VD constitute further limitations. Moreover, dietary calcium or sodium consumption was not controlled. Finally, the study was retrospective in nature with its inherent limitations. However, the strict inclusion and exclusion criteria, as well as the long follow-up time (median of 39 months), constitute the strengths of the study. Nevertheless, this is the first study that showed VDI urolithiasis patients treated with VDCS are at increased risk of de novo hypercalciuria and de novo stones. Future prospective randomized trials should be performed to assess the long-term lithogenic effects of VDCS in patients with a history of urolithiasis.

\section{Conclusion}

This study showed that combined VD and calcium supplementation in VDI urolithiasis patients resulted in significantly higher mean urinary calcium excretion. In addition, de novo hypercalciuria and stone development were associated with VDCS. Therefore, VDI patients with history of urolithiasis receiving VDCS are advised to follow-up with 24-hour urine collections and imaging studies to assess for de novo stone formation.

Acknowledgements: This work was supported in part by the Fond de Recherche en Santé du Québec (FRSQ) Research-Scholar Grant to Dr. Sero Andonian and the Health Professional Student Research Award from the Canadian Institute for Health Research to Charles Hesswani and The American Urological Association Urology Care Foundation Research Scholar Award and the CUASF-SIU International Scholarship to Dr. Yasser Noureldin.

Competing interests: The authors declare no competing financial or personal interests.

This paper has been peer-reviewed.

\section{References}

1. Mithal A, Wahl DA, Bonjour JP, et al. Global vitamin D status and determinants of hypovitaminosis D. Osteoporos Int 2009;20:1807-20. http://dx.doi.org/10.1007/s00198-009-0954-6

2. Kuchuk NO, van Schoor NM, Pluijm SM, et al. Vitamin D status, parathyroid function, bone turnover, and BMD in postmenopausal women with osteoporosis: Global perspective. J Bone Miner Res 2009:24:693701. http://dx.doi.org/10.1359/ibmr.081209

3. Holick MF. Vitamin D deficiency. N Engl J Med 2007;357:266-81. http://dx.doi.org/10.1056/ NEJMra070553

4. Elkoushy MA, Sabbagh R, Unikowsky B, et al. Prevalence and metabolic abnormalities of vitamin D-inadequate patients presenting with urolithiasis to a tertiary stone clinic. Urology 2012;79:781-5. http:// dx.doi.org/10.1016/i.urology.2011.09.004

5. Jackson RD, LaCroix AZ, Gass $M$, et al. Calcium plus vitamin $D$ supplementation and the risk of fractures. N Engl J Med 2006;354:669-83. http://dx.doi.org/10.1056/NEJMoa055218

6. Wallace RB, Wactawski-Wende J, O'Sullivan MJ, et al. Urinary tract stone occurrence in the Women's Health Initiative (WHI) randomized clinical trial of calcium and vitamin D supplements. Am I Clin Nutr 2011;94:270-7. http://dx.doi.org/10.3945/aicn.110.003350

7. Eisner BH, Thavaseelan $S$, Sheth $S$, et al. Relationship between serum vitamin $D$ and 24-hour urine calcium in patients with nephrolithiasis. Urology 2012;80:1007-10. http://dx.doi.org/10.1016/i. urology.2012.04.041 
Hesswani et al.

8. Leaf DE, Korets $\mathrm{R}$, Taylor $\mathrm{EN}$, et al. Effect of vitamin $\mathrm{D}$ repletion on urinary calcium excretion among kidney stone formers. Clin J Am Soc Nephrol 2012;7:829-34. http://dx.doi.org/10.2215/CJN.11331111

9. Berlin T, Emtestam L, Bjorkhem I. Studies on the relationship between vitamin D3 status and urinary excretion of calcium in healthy subjects: effects of increased levels of 25-hydroxyvitamin D3. Scand I Clin Lab Invest 1986;46:723-9. http://dx.doi.org/10.3109/00365518609084043

10. Taylor EN, Curhan $\mathrm{GC}$. Dietary calcium from dairy and nondairy sources, and risk of symptomatic kidney stones. J Urol 2013;190:1255-9. http://dx.doi.org/10.1016/i.juro.2013.03.074

11. Elkoushy MA, Jundi $M$, Lee TTN, et al. Bone mineral density status in urolithiasis patients with vitamin D inadequacy followed at a tertiary stone centre. Can Urol Assoc J 2014;8:323-8. http://dx.doi. org/10.5489/cuai.2055

12. Kramer HJM, Grodstein F, Stampfer MJ, et al. Menopause and postmenopausal hormone use and risk of incident kidney stones. J Am Soc Nephrol 2003;14:1272-7. http://dx.doi.org/10.1097/01. ASN.0000060682.25472.C3
13. Nayan M, Elkoushy MA, Andonian S. Variations between two 24-hour urine collections in patients presenting to a tertiary stone clinic. Can Urol Assoc J 2012;6:467-71.

14. Paterson R, Fernandez A, Razvi H, et al. Evaluation and medical management of the kidney stone patient. Can Urol Assoc J 2010;4:375-9. http://dx.doi.org/10.5489/cuaj.10166

15. Berlin T, Bjorkhem I, Collste L, et al. Relation between hypercalciuria and vitamin D3-status in patients with urolithiasis. Scand J Urol Nephrol 1982;16:269-73. http://dx.doi.org/10.3109/00365598209179765

16. Health Canada. http://www.hc-sc.gc.ca.

17. Bischoff-Ferrari HA, Giovannucci E, Willett WC, et al. Estimation of optimal serum concentrations of 25-hydroxyvitamin D for multiple health outcomes. Am J Clin Nutr 2006;84:18-28.

Correspondence: Dr. Sero Andonian, Associate Professor of Urology, McGill University Health Centre, 1001 Boul Decarie, Suite D05.5331, Montreal, QC H4A 3J1; sero.andonian@muhc.mcgill.ca 